\title{
Europäische Zusammenarbeit in den Geisteswissenschaften
}

\author{
WOLFGANG J. MOMMSEN \\ (Heinrich-Heine-Universität Düsseldorf)
}

Ich komme gern der Aufforderung auf, anläßlich der Begründung des Instituts für die Geschichte der europäischen Expansion hier das Wort zu ergreifen und einige Reflexionen über die Zusammenarbeit auf dem Felde der Geisteswissenschaften und insbesondere der Geschichtswissenschaft vorzutragen. Ich tue dies umso lieber, als ich die Notwendigkeit einer Intensivierung der Kooperation der europäischen Nationen auf wissenschaftlichem Felde zu einem Zeitpunkt, an welchem wir vor der Schwelle der politischen und wirtschaftlichen Einheit Europas stehen, für unabweisbar halte.

Der grosse niederländische Historiker Johan Huizinga hat einmal die besondere Befähigung, zugleich aber die besondere Berufung des holländischen Volkes, angesichts seiner Offenheit sowohl gegenüber der angelsächsischen wie auch der romanischen und dem deutschen Kulturtradition für eine Mittlerstellung zwischen West- und Mitteleuropa hervorgehoben. In diesem Sinne erscheint mir die Gründung eines solchen Instituts gerade in Leiden, einer Universität, deren traditionsreiche Geschichte in eine Zeit zurückreicht, in der Wissenschaft eine kosmopolitische oder, genauer betrachtet, eine wesentlich europäische Orientierung besaß, besonders angemessen. Der Historiographiehistoriker erinnert sich zudem, daß, gewiß nicht zufällig, der erste internationale Historikertag im Jahre 1898 in Den Haag zusammentrat, unter dem Vorsitz des damaligen holländischen Außenministers Willem de Beaufort, als eines Ablegers der Société d'histoire diplomatique. Dies darf als gutes Omen gelten.

Wissenschaft ist seit der Entstehung eines modernen Wissenschaftsverständnisses im 17. und frühen 18 . Jahrhundert ihrem.inneren Wesen nach stets international ausgerichtet gewesen; jedoch wird man zugleich sagen müssen, daß diese ihre Bestimmung in den vergangenen zwei Jahrhunderten, nach dem Verblassen der großen universalistischen Bewegung der Aufklärung, immer wieder in den Schatten nationalistischer Bestrebungen und nationalistischer Vorurteile geraten ist. Die Geschichtswissenschaften haben sich allzu häufig zur Dienerin jeweils politischer und ideologischer Bedürfnisse machen lassen, vornehmlich zum Zwecke der Stiftung natio- 
naler Identitäten oder der Legitimierung politischer Systeme oder politischer Bewegungen. Insoweit haben wir es auf dem Gebiet der Geisteswissenschaften - und dies gilt natürlich auch für die Geschichtswissenschaft - mit widerstreitenden Tendenzen zu tun.

Ungeachtet des prinzipiellen Bekenntnisses zur Internationalität der Wissenschaft waren die Geistes- und Sozialwissenschaften im 19. und in der ersten Hälfte des 20. Jahrhunderts sehr stark in die jeweiligen nationalen Kulturen eingebunden. Max Weber beispielsweise bekannte in seiner berühmten Freiburger Antrittsrede, obschon diese in mancher Hinsicht bereits das später von ihm mit Leidenschaft verfochtene Prinzip der Werturteilsfreiheit der Wissenschaft vorwegnahm: 'Die Volkswirtschaftslehre als erklärende und analysierende Wissenschaft is international, allein sobald sie Werturteile fältt, ist sie gebunden an diejenige Ausprägung des Menschentums, die wir in unserem eigenen Wesen finden.' 'Weber plädierte demnach mit Entschiedenheit für einen nationalistischen 'Bewertungsmaßstab' in der Volkswirtschaftslehre und, so dürfen wir folgern, im Prinzip auch in den Kulturwissenschaften. Das galt in Europa ganz allgemein. Es verwundert nicht, wenn beispielsweise die Historiker an der Universität Berlin 1908 gegen die Durchführung eines Internationalen Kongresses der Geschichtswissenschaft Berlin eindwandten, daß 'die großen Macht- und Völkerkämpfe', mit denen es die politische Geschichte in erster Linie zu tun habe, nur auf nationaler Grundlage behandelt werden könnten und in diesen Fragen 'ein gemeinsamer Boden für die internationale Verständigung' nicht gefunden werden könnte." Freilich gab es auch andere Meinungen. Theodor Mommsen beispielsweise bekannte 1901 im Zusammenhang der Vorbereitungen für den Internationalen Historikertag in Rom 1903: 'Die Hoffnung meiner Jugend: die gegenwärtige Zivilisation vereint $\mathbf{z u}$ sehen, um die gewesene zu studieren und in den Grenzen meines Talentes zu diesem unsterblichen Werke beizutragen, ist vielleicht nicht ganz Traum und Illusion."'"

Wir wissen, daß die Entwicklung der Dinge zunächst in eine entgegengesetzte Richtung gegangen ist. Insbesondere die Geisteswissenschaften wurden in den Strudel der nationalistischen Strömungen der Epoche hineingerissen. Sie wurden bemüht vornehmlich zur Stiftung nationaler Identität und zur Legitimierung des jeweils eigenen politischen und gesellschaftlichen Systems. Sie gerieten demgemäß selbst noch stärker in das Fahrwasser zeitgenössischer nationaler Vorurteile, als dies ohnehin schon der Fall gewesen war. Die Bemühungen der Wissenschaft der kleineren europäischen Nationen, vornehmlich der Schweizer, der Belgier, der Norweger und Schweden und nicht zuletzt der Niederländer, dem entgegenzuwirken, stießen weithin auf taube Ohren, auch wenn es an Gegentendenzen nicht gefehlt hat. Ihren Höhepunkt fand die Entfremdung zwischen den Geistesund Sozialwissenschaften der europäischen Nationen im Ersten Weltkriege. Die Wissenschaftler und Intellektuellen in allen kriegführenden Staaten stellten sich, mit nur wenigen Ausnahmen, vorbehaltlos hinter die kriegs- 
anstrengungen der eigenen Nation, allen voran die deutsche Wissenschaft. Sie glaubte im Ersten Weltkriege die Auseinandersetzung zwischen mehreren grundverschiedenen, einander radikal entgegengesetzten Weltkulturen zu sehen und gab so dem imperialistischen Völkerringen eine ganz unangemessene geistige Überhōhung. Am 4. Oktober 1914 traten 93 deutsche Akademiker mit einem 'Aufruf an die Kulturwelt' hervor, der die deutschen Kriegsanstrengungen als berechtigte Verteidigung der deutschen Kultur gegen die 'russischen Horden' und deren Helfershelfer in England und Frankreich rechtfertigte. ${ }^{+}$Umgekehrt vermeinte der französische Mathematiker Paul Painlevé 1918, daß die deutsche Wissenschaft ein einziges 'riesiges Unternehmen' gewesen sei, 'in dem ein ganzes volk mit geduldiger Servilität an der Herstellung der schrecklichsten Mordmaschinerie arbeitete, die je existierte'. Jene, die diesem Strom nationalistischer Exzesse auch im Felde der internationalen Wissenschaften Einhalt zu gebieten versuchten, wie in Deutschland Ernst Troeltsch oder in England James T. Shotwell, vermochten erst nach Ende des Ersten Weltkrieges mit ihrer Botschaft Gehör zu finden.

Die Versuche in der Zwischenkriegszeit, die zerrissenen Bande zwischen der deutschen und der westeuropäischen Wissenschaft wider neu zu begründen, können hier nicht im einzelnen geschildert werden. Unübersehbar freilich ist, daß sie gegen die extremen nationalistischen und schließlich die fascistischen bestrebungen der Zeit am Ende keinerlei Chance hatte. Vielmehr gipfelte die Entwicklung in dem erzwungenen Exodus einer groBen Zahl deutscher Wissenschaftler nich allein Jüdischen Glaubens, späterhin gefolgt von der Unterdrückung und teilweise der Verfolgung auch der Wissenschaftler in den vom nationalsozialistischen Deutschland eroberten Ländern. Die Indienstnahme der Geistes- und Sozialwissenschaften durch die Faschismen markierte den Höhepunkt der nationalistischen Verfremdung der kosmopolitischen Traditionen der Wissenschaften vom Menschen seit dem 18. Jahrhundert.

Unter dem Eindruck der Machtergreifung des Nationalsozialismus meinten manche Beobachter, wie Julien Benda, der in seinem Werk 'La Trahison des Clercs' aus diesen offenbaren Fehlentwicklungen der Zeit radikale folgerungen gezogen hatte, daß nur eine radikale Einebnung der unterschiedlichen nationalistischen Traditionen der europäischen Völker zugunsten der Schaffung einer einzigen europäischen Nation, in Anknüpfung an die kosmopolitische Tradition der Aufklärung, werde Abhilfe bringen können. Damals trat ihm Johan Huizinga öfentlich entgegen. Er sprach sich gegen eine egalitäre Einebnung der unterschiedlichen Nationalkulturen, die miteinander konkurrieren, aus und plädierte in Gegenteil für die Anerkennung ihres je eigenen Beitrags zu einer gemeinsamen europäischen Kultur. Er bestimmte die wesentliche Aufgabe geisteswissenschaftlichen und speziell historiographischen Bemühens in folgender Weise: 'Anerkennung des Fremden, das doch Fremdes bleibt, Einfühlung in dessen Geist, wăhrend man den eigenen behauptet, das sind die Fähigkeiten, 
welche sämtliche Nationen der Welt noch in einer langen Schulzeit zu üben haben werden.' ${ }^{\prime i}$ In den 30 er Jahren bestand in dieser Hinsicht wenig Grund zum Optimismus, die großen Exzesse der Faschismen und zugleich jene des Stalinismus, die beide auf ihre Weise eine einebnung der reichen kulturellen Traditionen Europas zugunsten von engstirnigen Ideologien betrieben, lagen noch in der Zukunft. Dennoch hat sich Johan Huizingas Prognose aus dem Jahre 1933 am Ende dennoch bewahrheitet: 'Die nationale Erbitterung, die edle Geister in den Wahnsinn getrieben hat, wird letzten Endes dem europäischen Gedanken dienen. Das Fieber wird fallen. Die großen Irrtümer sind noch immer überwunden worden.'

Heute stehen wir an der Schwelle einer neuen Epoche Europas und der Welt. Nicht nur sind die Schrecken des Zweiten Weltkrieges verblaßt, wenn auch nicht vergessen und die ideologischen Postulate der Faschismen gründlich entzaubert, sondern auch der sog. 'reale Sozialismus' des marxistisch-leninistischen 'Systems zusammengebrochen, der die historische Wahrheit zugunsten einer herrschenden bürokratischen Klasse von kommunistischen Funktionären und deren Helfershelfern je nach Bedarf beliebig verfälschte. Eine 'dritte demokratische Revolution', die ihren letzten großen Höhepunkt mit dem Fall der 'Mauer' am 9. November 1989 fand, hat über das sowjetische imperiale System gesiegt. Wir stehen am Anfang einer neuen Epoche, in der endlich die Voraussetzungen für freie Kooperation der Wissenschaft, und gerade auch der Geistes- und Sozialwissenschaften, in einem bisher nicht erreichbarem Maße gegeben sind. Es kommt hinzu, daß wir an der Schwelle der Schaffung einer gemeinsamen Ordnung Europas stehen, welche die bisherigen Nationalsouveränitäten transzendiert. In diesem Zusammenhang kommt insbesondere den Geisteswissenschaften und der Geschichtswissenschaft die Aufgabe zu, die Gemeinsamkeiten der kulturellen Traditionen Europas in das Bewußtsein der breiteren Öffentlichkeit zu rücken, unbeschadet der unterschiedlichen nationalen Eigenarten der europäischen Völker.

In einer solchen Situation ist es besonders zu begrüßèn, daß Institute wie das hier zu begründende es sich als Aufgabe setzen, ihre Forschungen grundsätzlich auf europäischer Grundlage zu betreiben. Dieser Aufgabe hat sich seit einigen Jahren auch die European Science Foundation in Straßburg zugewandt und ein Netzwerk europäischer Zusammenarbeit auf dem Felde der geistes- und sozialwissenschaftlichen Forschung begründet, ein Bemühen, an dem gerade die Universität Leiden auf dem Teilgebiet der europäischen Expansion maßgeblich mitgewirkt hat. Aller Voraussicht nach wird das größte Problem der vor uns liegenden Jahrzehnte das NordSud-Verhältnis sein, nachdem der Ost-West-Gegensatz gleichsam seine Schärfe verloren hat und die Entwicklungen auf internationalem Felde nicht länger dominierend überlagert. Insofern wächst der Erforschung der Expansion Europas über den gesamten Erdball, dessen Folgen die heutige Weltsituation in großem Maße bestimmen, vermehrte Bedeutung zu. Herkömmlicherweise wurden der Kolonialismus und der Imperialismus sowie 
seine Folgewirkungen auch nach dem Ende der Ära der Dekolonisation ganz überwiegend innerhalb nationaler Abgrenzungen wissenschaftlich untersucht; die Engländer studierten vorzugsweise die Geschichte der Territorien des britischen empire, die Franzosen die Geschichte des französischen Kolonialreiches, die Deutschen die Geschichte ihrer Kolonien und die Holländer vornehmlich das Schicksal ihrer ehemaligen Kolonialbesitzungen im Fernen Osten. Dies ist heute, in einer Situation, in der peripherieorientierte Fragestellungen vorherrschen, zunehmend fragwürdig geworden; die europäische Expansion mit ihren säkularen Auswirkungen auf die gegenwärtige Welt wurde von den betroffenen Völkern nicht in erster Linie als eine britische, französische, deutsche oder niederländische wahrgenommen, sondern als eine europäische, als die Einführung, ja Oktroyierung europäischer Herrschaft, als gewaltsame Öffnung der betreffenden Territorien für den von den Industriestaaten Europas dominierten Weltmarkt, als den Transfer europäischer Kultur, europäischer Wissenschaft und europäischer Technologie oft auf Kosten des Absterbens indigener kultureller Traditionen. Davon abgesehen hielt sich die Aktivität der Europäer vor Ort, sei es der Missionare, sei es der Pflanzer und Kaufleute, nur selten an nationale Grenzen; das wirtschaftliche Engagement deutscher Wirtschaftsunternehmungen in den Territorien des britischen Empire dürfte beispielsweise um ein vielfaches größer gewesen sein als das Engagement in den deutschen Kolonien selbst; umgekehrt dürfte aller Wahrscheinlichkeit nach, wenn auch in etwas geringerem Maße, das Gleiche gelten. Alles spricht demgemäß dafür, gerade auch auf dem Gebiet der Erforschung der Geschichte der europäischen Expansion, an die Stelle der überholten nationalgeschichtlichen Forschungsansätze, die überdies viel zu sehr die Bedingungen in den Metropolen und die Tätigkeit der Kolonialverwaltungen zum Gegenstand der Analyse erkoren haben, gesamteuropäisch orientierte Forschungsstrategien zu setzen. Dafür wird das Institut für die Geschichte der Europäischen Expansion und der Reaktionen der indigenen Bevölkerung, eine hervorragende Grundlage abgeben, nicht zuletzt dank seiner Einbettung in ein akademisches Umfeld, in dem Experten für Sprache, Kultur und Geschichte der Völker der Dritten Welt in bemerkenswerter Dichte zur Verfügung stehen. Es ist zu hoffen, daß es in Zukunft den Nukleus einer europäischen Forschersgruppe abgeben wird, die sich diesen Aufgaben in gemeinsamer Arbeit zuwendet. 


\section{Anmerkungen}

1 Max Weber, Gesammte Politische Schriften (3. Auflage; Tübingen 1971) 19.

2 Karl Dietrich Erdmann, Die Ökumene der Historiker. Geschichte der Internationalen Historikerkongresse und des Comité International des Sriences Historiques (Göttingen 1987) 66.

3 Ibidem, 42.

4 Vgl. Hermann Kellerman ed., Der Krieg der Geister 1914 (Weimar 1915) 64-68.

5 Erdmann, Die Oekumene der Historiker, 98.

6 Johan Huizinga, 'Geschichte und Kultur' in: Gesammelte Aufsätze. K. Köster ed. (Stuttgart 1954) 355.

7 Ibidem, 373. 